\title{
Characterization of Strongly Exposed Points in General Köthe-Bochner Banach Spaces
}

by

\author{
Houcine BENABDELLAH and My Hachem LALAOUI RHALI
}

Presented by Czestaw OLECH

Summary. We study strongly exposed points in general Köthe-Bochner Banach spaces $X(E)$. We first give a characterization of strongly exposed points of the set of $X$-selections of a measurable multifunction $\Gamma$. We then apply this result to the study of strongly exposed points of the closed unit ball of $X(E)$. Precisely we show that if an element $f$ is a strongly exposed point of $B_{X(E)}$, then $|f|$ is a strongly exposed point of $B_{X}$ and $f(\omega) /\|f(\omega)\|$ is a strongly exposed point of $B_{E}$ for $\mu$-almost all $\omega \in S(f)$.

1. Introduction. Preliminary results. Let $E$ be a separable Banach space with norm $\|\cdot\|$, and $(\Omega, \mathcal{F}, \mu)$ be a complete $\sigma$-finite measure space. A Köthe space $X$ over $(\Omega, \mathcal{F}, \mu)$ is an ideal of the vector lattice $L^{0}(\mu)$ of real measurable functions which is equipped with a monotone norm $\|\cdot\|_{X}$ (i.e. $\|f\|_{X} \leq\|g\|_{X}$ whenever $f, g \in X$ and $|f| \leq|g|$ ) for which it is a Banach space. The order dual $X^{\prime}$ associated to $X$ is the space of all functions $g$ in $L^{0}(\mu)$ such that $\operatorname{supp}(g) \subset \operatorname{supp}(X)$ and $f g \in L^{1}(\mu)$ for all $f$ in $X$. It is known that $X^{\prime}$ is a Banach space for the usual norm

$$
\|g\|_{X^{\prime}}:=\sup \left\{\int_{\Omega} f g d \mu: f \in X \text { and }\|f\|_{X} \leq 1\right\}, \quad g \in X^{\prime},
$$

and that $X^{\prime}$ is isometrically isomorphic to the topological dual $X^{*}$ if $X$ is order continuous.

Let us define the Köthe-Bochner Banach space $X(E)$ : given a vector function $f: \Omega \rightarrow E$, we denote by $|f|$ its modulus function defined by $\omega \mapsto$ $\|f(\omega)\|$. Let $X(E)$ be the vector space of all Bochner measurable functions

2000 Mathematics Subject Classification: Primary 05C38, 15A15; Secondary 05A15, $15 \mathrm{~A} 18$.

Key words and phrases: Köthe-Bochner Banach spaces, strongly extreme points, measurable selections. 
$f: \Omega \rightarrow E$ such that $|f|$ belongs to $X$. We will identify a function $f$ in $X(E)$ with its equivalence class under the relation of equality $\mu$-a.e. This leads to defining a norm in $X(E)$ by setting

$$
\|f\|_{X(E)}:=\||f|\|_{X}, \quad f \in X(E) .
$$

The space $X(E)$ is a Banach space for this norm.

For any vector function $f: \Omega \rightarrow E$, we define the support $S(f)$ of $f$ to be the set of all $\omega$ in $\Omega$ such that $f(\omega) \neq 0$.

Let $K$ be a nonempty closed convex subset of $E$. We say that a point $x_{0} \in K$ is a strongly exposed point of $K$ if there exists a linear form $x^{*} \in E^{*}$ which strongly exposes $K$ at $x$, that is, $\sup x^{*}(K)=x^{*}\left(x_{0}\right)$ and, whenever $\left\{x_{n}\right\} \subset K$ and $\lim _{n \rightarrow \infty} x^{*}\left(x_{n}\right)=x^{*}(x)$, then $\lim _{n \rightarrow \infty}\left\|x_{n}-x\right\|=0$. We will denote by $\operatorname{Str}-\exp (K)$ the set (possibly empty) of all strongly exposed points of $K$.

Strongly exposed points can be described in terms of slices. For $K \subset E$, the slice of $K$ determined by the functional $x^{*}$ in $E^{*}$ and $\delta>0$ is the subset of $K$ given by

$$
S\left(x^{*}, K, \delta\right)=\left\{x \in K: x^{*}(x)>\sup x^{*}(K)-\delta\right\} .
$$

In [8], $\mathrm{Hu}$ and B. L. Lin studied strongly exposed points in $L^{p}$-spaces and proved that if $f \in L^{p}(\mu, X),\|f\|=1$ and $f(t) /\|f(t)\| \in \operatorname{Str}-\exp \left(B_{X}\right)$ for almost all $t \in \operatorname{supp} f$, then $f \in \operatorname{Str}-\exp \left(B_{L^{p}(\mu, X)}\right)$. In 1998 P. K. Lin and Sun [13] showed that for an order continuous Köthe Banach space $X$ over $(\Omega, \mathcal{F}, \mu)$, if an element $f$ of the closed unit ball $B_{X(E)}$ of $X(E)$ satisfies the conditions:

(i) $|f|$ is a strongly exposed point of $B_{X}$,

(ii) $f(\omega) /\|f(\omega)\| \in \operatorname{Str}-\exp \left(B_{E}\right)$ for $\mu$-almost all $\omega \in S(f)$,

then $f$ is a strongly exposed point of $B_{X(E)}$.

In this paper, we first give a characterization of strongly exposed points of the set of $X$-selections of a measurable multifuction $\Gamma$ and we show that the converse of the result of [13] is also true, i.e. if $f$ is a strongly exposed point of $B_{X(E)}$ then the conditions (i) and (ii) are satisfied.

2. Preliminary lemmas. Let us recall the following lemmas which provide some nice characterizations and properties of strongly exposed points.

Lemma 2.1. Let $K$ be a closed convex subset of $E, x_{0} \in K$ and $x^{*} \in E^{*}$. The following assertions are equivalent:

(i) $x^{*}$ strongly exposes $K$ at $x_{0}$.

(ii) For any $\varepsilon>0$, there exists $\delta>0$ such that $\operatorname{diam} S\left(x^{*}, K, \delta\right)<\varepsilon$ and $x_{0} \in S\left(x^{*}, K, \delta\right)$, where diam $S$ stands for the diameter of a subset $S$ of $E$. 
(iii) $\sup x^{*}(K)=x^{*}\left(x_{0}\right)$ and for any $\varepsilon>0$, there exists $\delta>0$ such that for all $x \in K$, the condition $x^{*}(x)>x^{*}\left(x_{0}\right)-\delta$ implies $\left\|x-x_{0}\right\|<\varepsilon$.

Lemma 2.2. Let $K_{0}$ be a convex closed subset of $E$ and $x_{0} \in K_{0} \subset K$. If $x^{*}$ strongly exposes $K$ at $x_{0}$, then $x^{*}$ strongly exposes $K_{0}$ at $x_{0}$.

The following classical lemma gives conditions under which strong convergence in $L^{1}$ follows from weak convergence.

LEMMA 2.3. Let $\left(f_{n}\right) \subset L^{1}(\mu)$ and $f \in L^{1}(\mu)$ be such that $f_{n} \rightarrow f$ for $\sigma\left(L^{1}, L^{\infty}\right)$ and $f(t) \leq \liminf _{n \rightarrow \infty} f_{n}(t) \mu$-a.e. Then $\lim _{n \rightarrow \infty}\left\|f_{n}-f\right\|_{1}=0$.

We will also need the following theorem which is an extension of the classical Riesz representation theorem to the case of $X(E)^{*}$. For a short proof see [15].

TheOREM 2.1. Suppose that the Köthe space $X$ is order continuous. Then, for any $T \in X(E)^{*}$, there exists a weak ${ }^{*}$ measurable function $g$ : $\Omega \rightarrow E^{*}$ such that:

(i) The function $|g|: t \mapsto\|g(t)\|$ is measurable and belongs to $X^{\prime}$.

(ii) For any $f \in X(E), T(f)=\int_{\Omega}\langle g, f\rangle d \mu$.

(iii) $\|T\|=\|\mid g\|_{X^{\prime}}$.

We denote by $\operatorname{cc}(E)$ the set of all nonempty closed convex subsets of $E$. Let $\Gamma: \Omega \rightarrow \operatorname{cc}(E)$ be a given multifunction.

We shall say that the multifunction $\Gamma$ is graph-measurable if its graph $\operatorname{gr}(\Gamma):=\{(\omega, x) \in \Omega \times E: x \in \Gamma(\omega)\}$ belongs to $\mathcal{F} \otimes \mathcal{B}(E)$, where $\mathcal{B}(E)$ is the Borel tribe of $E$. A selection or section of $\Gamma$ is a function $\sigma: \Omega \rightarrow E$ such that $\sigma(\omega) \in \Gamma(\omega)$ for every $\omega \in \Omega$. It is well known (cf. [3], [7]) that if $\Gamma$ is graph-measurable, then $\Gamma$ admits a measurable selection. We shall denote by $L_{\Gamma}^{0}(\mu)$ the set of all measurable selections of $\Gamma$.

Let $X$ be a Köthe Banach space over $(\Omega, \Sigma, \mu)$. We shall say that the multifunction $\Gamma: \Omega \rightarrow \operatorname{cc}(E)$ is $X$-bounded if there exists a function $g \in X_{+}$ such that $\Gamma(\omega) \subset g(\omega) B_{E} \mu$-a.e., where $B_{E}$ is the closed unit ball of $E$.

Denote by $L_{\Gamma}^{X}(\mu)$ the set of all selections $\sigma$ of $\Gamma$ such that $\sigma \in X(E)$ (that is, $\left.L_{\Gamma}^{X}(\mu)=X(E) \cap L_{\Gamma}^{0}(\mu)\right)$. It is clear that if $\Gamma$ is graph-measurable and $X$-bounded, then $L_{\Gamma}^{X}(\mu)$ is a nonempty closed convex subset of $X(E)$.

In case $X=L^{1}(\mu)$, we speak of integrably bounded multifunctions instead of $X$-bounded multifunctions, and write $L_{\Gamma}^{1}(\mu)$ instead of $L_{\Gamma}^{X}(\mu)$.

3. The main results. We begin this section by stating the following characterization of strongly exposed selections in $X(E)$ of a measurable multifunction. Similar results in the case of denting points are given in [1] and $[2]$. 
TheOrem 3.1. Let $\Gamma: \Omega \rightarrow \operatorname{cc}(E)$ be a graph-measurable and $X$-bounded multifunction. Assume that $f \in L_{\Gamma}^{X}(\mu)$. Then $f$ is a strongly exposed point of $L_{\Gamma}^{X}(\mu)$ if and only if $f(\omega)$ is a strongly exposed point of $\Gamma(\omega)$ for almost all $\omega \in \Omega$.

Proof. By considering $\Gamma^{\prime}(\omega)=\Gamma(\omega)-f(\omega)$, it suffices to prove the result for $f=0$. We can also suppose that $\mu$ is a probability measure and that $\operatorname{supp}(X)=\Omega$. Let then $\beta$ in $X^{\prime}$ be such that $\|\beta\|_{X^{\prime}}=1$ and $\beta(\omega)>0$ for all $\omega \in \Omega$. We also assume that $\Gamma(\omega) \subset \alpha(\omega) B_{E} \mu$-a.e., where $\alpha \in X_{+}$.

By assumption, we have $0 \in \operatorname{Str}-\exp \Gamma(\omega) \mu$-a.e. Let us prove that $0 \in$ Str-exp $L_{\Gamma}^{X}(\mu)$. For $p \geq 1$ we put $\Omega_{p}:=\{x \in \Omega: p-1 \leq \beta(x)<p\}$. Then we get a measurable partition $\left\{\Omega_{p}\right\}_{p \geq 1}$ of $\Omega$. Denote by $p B_{E^{*}}^{\sigma}$ the closed ball $p B_{E^{*}}$ of $E^{*}$ equipped with the weak* topology, and recall that $p B_{E^{*}}^{\sigma}$ is a compact metric space. Let $\left\{\sigma_{n}\right\}_{n \geq 0}$ be a Castaing representation of the multifunction $\Gamma$. For every $n$, the function $\gamma_{n}^{p}: \Omega \times p B_{E^{*}}^{\sigma} \rightarrow \mathbb{R},\left(\omega, x^{*}\right) \mapsto$ $x^{*}\left(\sigma_{n}(\omega)\right)$, is $\mathcal{F} \otimes \mathcal{B}\left(p B_{E^{*}}^{\sigma}\right)$-measurable. It follows that the function $\gamma^{p}: \Omega \times$ $p B_{E^{*}}^{\sigma} \rightarrow \mathbb{R},\left(\omega, x^{*}\right) \mapsto \sup x^{*}(\Gamma(\omega))=\sup _{n} \gamma_{n}^{p}\left(\omega, x^{*}\right)$, is also $\mathcal{F} \otimes \mathcal{B}\left(p B_{E^{*}}^{\sigma}\right)$ measurable. Consider the multifunction $\Sigma_{p}: \Omega_{p} \rightrightarrows p B_{E^{*}}^{\sigma}$ defined by

$$
\begin{aligned}
\Sigma_{p}(\omega):=\left\{x^{*} \in E^{*}:\right. & \left\|x^{*}\right\| \leq \beta(\omega) \text { and } \\
& \forall \varepsilon \in \mathbb{Q}_{+}^{*}, \exists \delta \in \mathbb{Q}_{+}^{*} \text { such that } \sup x^{*}(\Gamma(\omega))<\delta \text { and } \\
& \left.\forall x \in \Gamma(\omega),\left(x^{*}(x)>\sup x^{*}(\Gamma(\omega))-\delta \Rightarrow\|x\| \leq \varepsilon\right)\right\} .
\end{aligned}
$$

From our hypothesis and Lemma 2.1, it is clear that for almost all $\omega \in \Omega_{p}$, the set $\Sigma_{p}(\omega)$ is nonempty. Moreover it is easy to check that

$$
\operatorname{gr}\left(\Sigma_{p}\right)=\bigcap_{\varepsilon \in \mathbb{Q}_{+}^{*}} \bigcup_{\delta \in \mathbb{Q}_{+}^{*}} \Phi_{p}(\varepsilon, \delta),
$$

where

$$
\begin{gathered}
\Phi_{p}(\varepsilon, \delta):=\left\{\left(\omega, x^{*}\right) \in \Omega \times p B_{E^{*}}^{\sigma}:\left\|x^{*}\right\| \leq \beta(\omega), \gamma\left(\omega, x^{*}\right)<\delta \text { and } \forall n \geq 0,\right. \\
\left.\left(x^{*}\left(\sigma_{n}(\omega)\right)>\gamma\left(\omega, x^{*}\right)-\delta \Rightarrow\left\|\sigma_{n}(\omega)\right\| \leq \varepsilon\right)\right\} .
\end{gathered}
$$

It follows that $\operatorname{gr}\left(\Sigma_{p}\right)$ belongs to $\mathcal{F} \otimes \mathcal{B}\left(p B_{E^{*}}^{\sigma}\right)$. By the Aumann selection theorem ([3, Chap. III $]$ ), we can choose a measurable selection $h_{p}: \Omega_{p} \rightarrow$ $p B_{E^{*}}^{\sigma}$ of the multifunction $\Sigma_{p}$. Putting $h(\omega):=h_{p}(\omega)$ for all $p \geq 1$ and $\omega \in \Omega_{p}$, we get a weak* measurable function $h$ from $\Omega$ to $E^{*}$ such that $\mu$-a.e., $h(\omega)$ strongly exposes $\Gamma(\omega)$ at 0 . Moreover, $|h|$ belongs to $X^{\prime}$ since by construction $\|h(\omega)\| \leq \beta(\omega)$ on $\Omega$. Consider the continuous linear form $h^{*}$ on $X(E)$, defined by

$$
h^{*}(f):=\int_{\Omega} h(\omega)(f(\omega)) \mu(d \omega), \quad f \in X(E) .
$$

To complete the proof of the direct part of our theorem we shall prove that

$$
\sup h^{*}(X(E))=0 \text {. }
$$


(B) For every sequence $\left(g_{n}\right)$ in $L_{\Gamma}^{X}(\mu)$,

$$
\lim _{n \rightarrow \infty} h^{*}\left(g_{n}\right)=0 \quad \text { implies } \quad \lim _{n \rightarrow \infty}\left\|g_{n}\right\|_{X(E)}=0 \text {. }
$$

Thus $h^{*}$ strongly exposes the closed convex set $L_{\Gamma}^{X}(\mu)$ at 0 .

Let us prove (A). Since $\mu$-a.e., $h(\omega)$ strongly exposes $\Gamma(\omega)$ at 0 , we have

$$
\sup h(\omega)(\Gamma(\omega))=0 \quad \mu \text {-a.e. }
$$

Hence for every $g \in L_{\Gamma}^{X}(\mu)$,

$$
\int_{\Omega} h(\omega)(g(\omega)) \mu(d \omega) \leq 0 .
$$

Thus sup $h^{*}\left(L_{\Gamma}^{X}(\mu)\right) \leq 0$. As $0 \in L_{\Gamma}^{X}(\mu)$, we conclude that $\sup h^{*}\left(L_{\Gamma}^{X}(\mu)\right)=0$.

Let us prove $(\mathrm{B})$. Assume that $\left(g_{n}\right)$ does not converge strongly to 0 in $X(E)$. Then we may suppose that, along a subsequence, $\left\|g_{n}\right\|_{X(E)} \geq \eta$ for some $\eta>0$. Notice now that the sequence $\left(\left\langle h, g_{n}\right\rangle\right)_{n \geq 0}$ of scalar functions is uniformly integrable in $L^{1}(\mu)$ since $\left|\left\langle h(\omega), g_{n}(\omega)\right\rangle\right| \leq \alpha(\omega) \mu$-a.e. It follows that it is relatively weakly compact in $L^{1}(\mu)$. By the Eberlein-Smulian theorem we may assume that, along a subsequence, $\left\langle h, g_{n}\right\rangle \rightarrow \phi$ weakly in $L^{1}(\mu)$ for some $\phi$ in $L^{1}(\mu)$. Mazur's theorem yields a sequence of convex combinations $\widetilde{g}_{n} \in \operatorname{co}\left\{g_{k}: k \geq n\right\}, n \in \mathbb{N}$, such that

$$
\lim _{n \rightarrow \infty}\left\langle h(\omega), \widetilde{g}_{n}(\omega)\right\rangle=\phi(\omega) \quad \mu \text {-a.e. }
$$

Set $\widetilde{g}_{n}:=\sum_{i=n}^{\nu_{n}} \lambda_{i}^{n} g_{i}$, where $\nu_{n} \geq n, \lambda_{i}^{n} \geq 0$ and $\sum_{i=n}^{\nu_{n}} \lambda_{i}^{n}=1$. We have

$$
\left\langle h(\omega), \widetilde{g}_{n}(\omega)\right\rangle=\sum_{i=n}^{\nu_{n}} \lambda_{i}^{n}\left\langle h(\omega), g_{i}(\omega)\right\rangle \leq 0 \quad \mu \text {-a.e. }
$$

Hence

$$
\phi(\omega) \leq 0 \quad \mu \text {-a.e. }
$$

On the other hand, by assumption we have $\int_{\Omega}\left\langle h, g_{n}\right\rangle d \mu \rightarrow 0$ and $\int_{\Omega}\left\langle h, g_{n}\right\rangle d \mu$ $\rightarrow \int_{\Omega} \phi d \mu$ as $n \rightarrow \infty$. Hence $\int_{\Omega} \phi d \mu=0$ and from (3.1) it follows that $\phi(\omega)=0 \mu$-a.e. Thus

$$
\lim _{n \rightarrow \infty}\left\langle h(\omega), \widetilde{g}_{n}(\omega)\right\rangle=0 \quad \mu \text {-a.e. }
$$

Now we have $\mu$-a.e.,

$$
\begin{aligned}
0 \geq \limsup _{n \rightarrow \infty}\left\langle h(\omega), g_{n}(\omega)\right\rangle & \geq \limsup _{n \rightarrow \infty} \sum_{i=n}^{\nu_{n}} \lambda_{i}^{n}\left\langle h(\omega), g_{i}(\omega)\right\rangle \\
& =\lim _{n \rightarrow \infty}\left\langle h(\omega), \widetilde{g}_{n}(\omega)\right\rangle=0 .
\end{aligned}
$$

Hence

$$
\limsup _{n \rightarrow \infty}\left\langle h(\omega), g_{n}(\omega)\right\rangle=\liminf _{n \rightarrow \infty}-\left\langle h(\omega), g_{n}(\omega)\right\rangle=0 \quad \mu \text {-a.e. }
$$


Since $\left\langle h, g_{n}\right\rangle \rightarrow 0$ weakly in $L^{1}(\mu)$, we deduce by Lemma 2.3 that

$$
\lim _{n \rightarrow \infty} \int_{\Omega}\left|\left\langle h, g_{n}\right\rangle\right| d \mu=0 .
$$

Let $\left(g_{n_{k}}\right)$ be a subsequence of $\left(g_{n}\right)$ such that $\lim _{k \rightarrow \infty}\left\langle h(\omega), g_{n_{k}}(\omega)\right\rangle=0$ $\mu$-a.e. Since $\mu$-a.e. $h(\omega)$ strongly exposes $\Gamma(\omega)$ at 0 , we deduce that

$$
\lim _{k \rightarrow \infty}\left\|g_{n_{k}}(\omega)\right\|=0 \quad \mu \text {-a.e. }
$$

Since $X$ is order continuous we also deduce that $\left\|g_{n_{k}}\right\|_{X(E)} \rightarrow 0$ as $k \rightarrow \infty$. This contradicts the fact that $\left\|g_{n}\right\|_{X(E)} \geq \eta$ for $n \in \mathbb{N}$ and finishes the proof of the sufficiency part of the theorem.

Necessity. Assume that 0 is a strongly exposed point of $L_{\Gamma}^{X}(\mu)$. Without loss of generality we can assume that $1_{\Omega} \in X$. Indeed, there exists $\gamma \in X_{+}$ such that $\|\gamma\|_{X}=1$ and $\gamma>0$ on $\Omega$; then $X$ is isometrically isomorphic to the Köthe space $\left(X_{1},\|\cdot\|_{X_{1}}\right)$, where $X_{1}=(1 / \gamma) X$ and $\|g\|_{X_{1}}:=\|\gamma g\|_{X}$. Then, by Theorem 2.1, there exists a weak* measurable function $h: \Omega \rightarrow E^{*}$ such that $|h|$ belongs to $X^{\prime}$ and the linear form $\widetilde{h}: f \mapsto \int_{\Omega}\langle h, f\rangle d \mu$ strongly exposes the subset $L_{\Gamma}^{X}(\mu)$ of $X(E)$ at 0 . Given $\varepsilon>0$ there exists $\delta>0$ such that for every $g \in L_{\Gamma}^{X}(\mu)$,

$$
\int_{\Omega}\langle h, g\rangle d \mu>-\delta \Rightarrow\|g\|_{X(E)}<\varepsilon .
$$

Let us first prove that

$$
\sup h(\omega)(\Gamma(\omega))=0 \quad \mu \text {-a.e. }
$$

For $n \in \mathbb{N}$ and $\omega \in \Omega$, we set

$$
A_{n}(\omega):=\left\{x \in \Gamma(\omega): \sup h(\omega)(\Gamma(\omega))-2^{-n}<h(\omega)(x)\right\} .
$$

It is clear that the multifunction $A_{n}: \Omega \rightrightarrows E$ is nonempty-valued and its graph $\operatorname{gr}\left(A_{n}\right)$ belongs to $\mathcal{F} \otimes \mathcal{B}(E)$. Let $u_{n}: \Omega \rightarrow E$ be a measurable selection of $A_{n}$. We have

$$
\forall \omega \in \Omega, \quad u_{n}(\omega) \in \Gamma(\omega), \quad \sup h(\omega)(\Gamma(\omega))-2^{-n}<h(\omega)\left(u_{n}(\omega)\right) .
$$

Notice that the function $\gamma: \omega \mapsto \sup h(\omega)(\Gamma(\omega))$ is $\mu$-integrable since $\Gamma(\omega) \subset \alpha(\omega) B_{E} \mu$-a.e. Moreover

$$
\int_{\Omega} \gamma(\omega) \mu(d \omega)-2^{-n} \leq \int_{\Omega} h(\omega)\left(u_{n}(\omega)\right) \mu(d \omega) \leq \int_{\Omega} \gamma(\omega) \mu(d \omega)
$$

and letting $n \rightarrow \infty$, we get

$$
\lim _{n \rightarrow \infty} \int_{\Omega} h(\omega)\left(u_{n}(\omega)\right) \mu(d \omega)=\int_{\Omega} \gamma(\omega) \mu(d \omega) .
$$


On the other hand, as $u_{n} \in L_{\Gamma}^{X}(\mu)$ and $\sup \widetilde{h}\left(L_{\Gamma}^{X}(\mu)\right)=0$ we have

$$
0 \geq \int_{\Omega} h(\omega)\left(u_{n}(\omega)\right) \mu(d \omega)
$$

so by passing once again to the limit as $n \rightarrow \infty$, we get

$$
0 \geq \int_{\Omega} \sup h(\omega)(\Gamma(\omega)) \mu(d \omega) \geq \sup \widetilde{h}\left(L_{\Gamma}^{X}(\mu)\right)=0 .
$$

Hence

$$
\int_{\Omega} \sup h(\omega)(\Gamma(\omega)) \mu(d \omega)=0 .
$$

As $\sup h(\omega)(\Gamma(\omega)) \geq 0 \mu$-a.e., we deduce that $\sup h(\omega)(\Gamma(\omega))=0 \mu$-a.e.

To complete the proof of the theorem, we shall prove that

(C) For $\mu$-almost every $\omega \in \Omega, h(\omega)$ strongly exposes $\Gamma(\omega)$ at 0 .

Let $\widetilde{\Omega}$ denote the set of all $\omega \in \Omega$ such that $h(\omega)$ strongly exposes $\Gamma(\omega)$ at 0 . By (3.3), we have

$$
\begin{aligned}
\widetilde{\Omega}=\left\{\omega \in \Omega: \forall \varepsilon \in \mathbb{Q}_{+}^{*}, \quad \exists \delta \in\right. & \mathbb{Q}_{+}^{*} \text { such that } \\
& \forall x \in \Gamma(\omega),(h(\omega)(x)>-\delta \Rightarrow\|x\| \leq \varepsilon)\} .
\end{aligned}
$$

Using a Castaing representation of the multifunction $\Gamma$, we can prove, as in the sufficiency step, that $\widetilde{\Omega} \in \mathcal{F}$. Condition (C) will be proved if we establish that

$$
\text { For every } \varepsilon>0, \mu(\Omega \backslash \widetilde{\Omega}) \leq \varepsilon \text {. }
$$

Let $\varepsilon>0$. For $n \geq 1$, set

$$
\begin{aligned}
\Omega(\varepsilon, n):=\left\{\omega \in \Omega: \forall \delta \in \mathbb{Q}_{+}^{*},\right. & \exists x \in \Gamma(\omega) \text { such that } \\
& \left.h(\omega)(x)>-\delta \text { and }\|x\|>\varepsilon / 2^{n}\right\} .
\end{aligned}
$$

Using a Castaing representation of $\Gamma$, we can easily prove that $\Omega(\varepsilon, n) \in \mathcal{F}$. Moreover,

$$
\Omega \backslash \widetilde{\Omega} \subset \bigcup_{n=1}^{\infty} \Omega(\varepsilon, n) .
$$

Fix $\eta=\varepsilon / 2^{n}$. Since $\widetilde{h}$ strongly exposes $L_{\Gamma}^{X}(\mu)$ at 0 , there is $\delta_{0}>0$ such that

$$
\forall g \in L_{\Gamma}^{X}(\mu), \quad \int_{\Omega}\langle h, g\rangle d \mu>-\delta_{0} \Rightarrow\|g\|_{X(E)} \leq \eta^{2} .
$$

Set

$$
\Omega_{\eta}:=\left\{\omega \in \Omega: \exists x \in \Gamma(\omega) \backslash \eta B_{E} \text { such that } h(\omega)(x)>-\delta_{0}\right\} .
$$

It is clear that $\Omega_{\eta} \in \mathcal{F}$. Let us prove that

$$
\left\|\chi_{\Omega_{\eta}}\right\|_{X} \leq \eta \text {. }
$$


Assume otherwise. Then the set

$$
M(\omega):=\left\{x \in \Gamma(\omega) \backslash \eta B_{E}: h(\omega)(x)>-\delta_{0}\right\}
$$

is nonempty for every $\omega \in \Omega_{\eta}$. Moreover standard arguments shows that the multifunction $M: \Omega \rightrightarrows E$ has measurable graph in $\mathcal{F} \otimes \mathcal{B}(E)$. Let $v: \Omega_{\eta} \rightarrow E$ be a measurable function such that $v(\omega) \in M(\omega)$ for every $\omega \in \Omega_{\eta}$. Extend $v$ to $\Omega$ by setting $v(\omega):=0$ for $\omega \in \Omega \backslash \Omega_{\eta}$. Then $v \in L_{\Gamma}^{X}(\mu)$. Moreover

$$
\int_{\Omega}\langle h, v\rangle d \mu>\int_{\Omega_{\eta}}-\delta_{0} d \mu \geq-\delta_{0}
$$

(recall that $\mu(\Omega)=1$ !) and

$$
\|v\|_{X(E)}=\left\|\chi_{\Omega_{\eta}}|v|\right\|_{X(E)} \geq\left\|\eta \chi_{\Omega_{\eta}}\right\|_{X}>\eta^{2} .
$$

Hence $v \in L_{\Gamma}^{X}(\mu), \int_{\Omega}\langle h, v\rangle d \mu>-\delta_{0}$ and $\|v\|_{X(E)}>\eta^{2}$. But this contradicts (3.5). So condition (3.6) is proved.

Now we remark that $\Omega(\varepsilon, n) \subset \Omega_{\eta}$ and hence $\left\|\chi_{\Omega(\varepsilon, n)}\right\|_{X} \leq \eta=\varepsilon / 2^{n}$. Using (3.4) we get

$$
\left\|\chi_{\Omega \backslash \tilde{\Omega}}\right\|_{X} \leq \sum_{n=1}^{\infty}\left\|\chi_{\Omega(\varepsilon, n)}\right\|_{X} \leq \varepsilon .
$$

Since $\varepsilon>0$ is arbitrary we get $\chi_{\Omega \backslash \widetilde{\Omega}}=0$. This completes the proof of the theorem.

We are now able to state the following theorem.

Theorem 3.2. Suppose that $X$ is an order continuous Köthe Banach space over $(\Omega, \mathcal{F}, \mu)$. Let $f$ be in the closed unit ball $B_{X(E)}$ of $X(E)$. If $f$ is a strongly exposed point of $B_{X(E)}$, then:

(i) $|f|$ is a strongly exposed point of $B_{X}$;

(ii) $f(\omega) /\|f(\omega)\| \in \operatorname{Str}-\exp \left(B_{E}\right)$ for $\mu$-almost all $\omega \in S(f)$.

Proof. We may assume without loss of generality that $\operatorname{supp}(X)=\Omega$. Suppose that $f$ is a strongly exposed point of $B_{X(E)}$. To prove (i) and (ii), consider the multifunction $\Gamma$ defined on $\Omega$ by

$$
\Gamma(\omega):=\|f(\omega)\| B_{E} \quad \text { for } \omega \in \Omega .
$$

It is obvious that $L_{\Gamma}^{X}(\mu)$ is a closed convex subset of $B_{X(E)}$ and that $f$ lies in $L_{\Gamma}^{X}(\mu)$. Hence $f$ is also a strongly exposed point of $L_{\Gamma}^{X}(\mu)$. By Theorem 3.1, $f(\omega)$ is a strongly exposed point of $\|f(\omega)\| B_{E}$ for $\mu$-almost every $\omega \in \Omega$. Thus (ii) is satisfied.

Let us prove (i). First, by assumption, there exists a linear form $T$ in $X(E)^{*}$ which strongly exposes $B_{X(E)}$ at $f$. We can suppose that $\|T\|=1$. Let $g: \Omega \rightarrow E^{*}$ given by Theorem 2.1 be such that $T=\langle g, \cdot\rangle$. We will prove that the linear form $\bar{T}:=\langle|g|, \cdot\rangle$ strongly exposes $B_{X}$ at $|f|$. 
It is obvious that $\||g|\|_{X^{\prime}}=\|f\|_{X(E)}=1$. Moreover

$$
1=\sup T\left(B_{X(E)}\right)=T(f) \leq \int_{\Omega}|g||f| d \mu \leq 1
$$

Hence

$$
\bar{T}(|f|)=1=\|\bar{T}\|=\sup \bar{T}\left(B_{X}\right) .
$$

Let $\left(h_{n}\right)$ be a sequence in $B_{X}$ such that $\lim _{n \rightarrow \infty} \bar{T}\left(h_{n}\right)=1$. We will prove that $\left\||f|-h_{n}\right\|_{X} \rightarrow 0$ as $n \rightarrow \infty$. First assume that the functions $h_{n}$ are nonnegative. Note that

$$
|g|(\omega) h_{n}(\omega)=\sup _{u \in S_{E}}\left\langle g(\omega), h_{n}(\omega) u\right\rangle \quad \text { for } \omega \in \Omega,
$$

where $S_{E}$ is the unit sphere of $E$. Since the measure $\mu$ is $\sigma$-finite, let $\gamma: \Omega$ $\rightarrow \mathbb{R}_{+}$be a measurable function such that $\gamma(\omega)>0$ on $\Omega$ and $\int_{\Omega} \gamma d \mu=1$. By (3.7) the set

$$
\Phi_{n}(\omega):=\left\{u \in S_{E}:|g|(\omega) h_{n}(\omega)-2^{-n} \gamma(\omega) \leq\left\langle g(\omega), h_{n}(\omega) u\right\rangle\right\}
$$

is nonempty for all $\omega$ in $\Omega$. The multifunction $\omega \mapsto \Phi_{n}(\omega)$ from $\Omega$ to the closed bounded subsets of $E$ is obviously measurable. Let $u_{n}: \Omega \rightarrow S_{E}$ be a measurable selection of $\Phi_{n}$. Then

$$
\begin{aligned}
|g|(\omega) h_{n}(\omega)-2^{-n} \gamma(\omega) & \leq\left\langle g(\omega), h_{n}(\omega) u_{n}(\omega)\right\rangle, \\
\left\|u_{n}(\omega)\right\| & =1 \quad \text { for } \omega \in \Omega .
\end{aligned}
$$

Set $f_{n}(\omega):=h_{n}(\omega) u_{n}(\omega)$ for $\omega \in \Omega$. Then $\left(f_{n}\right)$ is a sequence in $X(E)$ such that $\left|f_{n}\right|=h_{n}$ and $\lim _{n \rightarrow \infty} T\left(f_{n}\right)=1$. Indeed, from (3.8) it is clear that

$$
\int_{\Omega}|g| h_{n} d \mu-2^{-n} \leq \int_{\Omega}\left\langle g, f_{n}\right\rangle d \mu
$$

so the result follows. Now, since $T$ strongly exposes $B_{X(E)}$ at $f$, we deduce that

$$
\lim _{n \rightarrow \infty}\left\|f-f_{n}\right\|_{X(E)}=0
$$

By remarking that

$$
|| f\left|-h_{n}\right| \leq\left|f-f_{n}\right|
$$

we finally deduce that $\lim _{n \rightarrow \infty}|||f|-h_{n} \|_{X}=0$.

Suppose now that the functions $h_{n}$ are arbitrary (not necessarily nonnegative). Then the condition $\lim _{n \rightarrow \infty} \bar{T}\left(h_{n}\right)=1$ implies obviously that

$$
\lim _{n \rightarrow \infty} \bar{T}\left(h_{n}^{+}\right)=\lim _{n \rightarrow \infty} \bar{T}\left(\left|h_{n}\right|\right)=1 \text {. }
$$

So applying the preceding step to the sequences $\left(h_{n}^{+}\right)$and $\left(\left|h_{n}\right|\right)$ we deduce that

$$
\lim _{n \rightarrow \infty}\left\||f|-h_{n}^{+}\right\|_{X}=\lim _{n \rightarrow \infty}\left\||f|-\left|h_{n}\right|\right\|_{X}=0
$$


Hence $h_{n}=2 h_{n}^{+}-\left|h_{n}\right|$ converges strongly in $X$ to $|f|$. This proves that $\bar{T}$ strongly exposes $B_{X}$ at $|f|$ and completes the proof of the theorem.

\section{References}

[1] H. Benabdellah, Contribution aux problèmes de convergence fort-faible, à la géométrie des espaces de Banach et aux inclusions différentielles, doctoral thesis, Univ. Montpellier II, 1991.

[2] —, Denting points in Bochner Banach ideal spaces X(E), J. Convex Anal. 6 (1999), 183-194.

[3] C. Castaing and M. Valadier, Convex Analysis and Measurable Multifunctions, Lecture Notes in Math. 580, Springer, Berlin, 1977.

[4] P. Greim, Strongly exposed points in Bochner $L^{p}$-spaces, Proc. Amer. Math. Soc. 88 (1983), 81-84.

[5] -, A note on strongly extreme and strongly exposed points in Bochner $L^{p}$-spaces, ibid. 93 (1985), 65-66.

[6] R. Grząślewicz, H. Hudzik and W. Kurc, Extreme and exposed points in Orlicz spaces, Canad. J. Math. 44 (1992), 505-515.

[7] C. J. Himmelberg, Measurable relations, Fund. Math. 87 (1975), 53-72.

[8] Z. B. Hu and B. L. Lin, Strongly exposed points in Lebesgue Bochner function spaces, Proc. Amer. Math. Soc. 120 (1994), 1159-1165.

[9] -, 一, Extremal structure of the unit ball of $L^{p}(\mu, X)^{*}$, J. Math. Anal. Appl. 200 (1996), 567-590.

[10] B. L. Lin and P. K. Lin, Denting points in Bochner $L^{p}$-spaces, Proc. Amer. Math. Soc. 97 (1986), 629-633.

[11] B. L. Lin, P. K. Lin and S. L. Troyanski, Characterizations of denting points, ibid. 102 (1988), 526-528.

[12] P. K. Lin and H. Y. Sun, Denting points in Köthe-Bochner function spaces, preprint.

[13] P. K. Lin and H. Y. Sun, Extremity in Köthe-Bochner function spaces, J. Math. Anal. Appl. 218 (1998), 136-154.

[14] J. Lindenstrauss and L. Tzafriri, Classical Banach Spaces II. Function Spaces, Ergeb. Math. Grenzgeb. 97, Springer, New York, 1979.

[15] M. Talagrand, Weak Cauchy sequences in $L^{1}(E)$, Amer. J. Math. 106 (1984), 703724 .

H. Benabdellah and M. H. Lalaoui Rhali

Department of Mathematics

University Cadi Ayyad

Faculty of Sciences Semlalia

P.O. Box 2390, Marrakesh, Morocco

E-mail: benabdellah@ucam.ac.ma

hmlalaoui@ucam.ac.ma 